\title{
IL-17-mediated mitochondrial dysfunction impairs apoptosis in rheumatoid arthritis synovial fibroblasts through activation of autophagy
}

\author{
Eun Kyung Kim ${ }^{1,2}$, Jeong-Eun Kwon ${ }^{1,2}$, Seon-Young Lee ${ }^{1,2}$, Eun-Jung Lee ${ }^{1,2}$, Da Som Kim ${ }^{1,2}$, Su-Jin Moon ${ }^{3}$, Jennifer Lee ${ }^{1,3}$, \\ Seung-Ki Kwok ${ }^{1,3}$, Sung-Hwan Park ${ }^{1,3}$ and Mi-La Cho ${ }^{*, 1,2}$
}

Fibroblast-like synoviocytes (FLSs) are a major cell population of the pannus that invades cartilage and bone in rheumatoid arthritis (RA). FLS resistance to apoptosis is a major characteristic of RA. The aims of this study were to investigate the effects of interleukin-17 (IL-17) and IL-17-producing T helper (Th17) cells on resistance to apoptosis in FLSs from RA patients (RA FLSs) and their roles in mitochondrial dysfunction and autophagy. Mitochondrial function was assessed in RA FLSs and FLSs from osteoarthritis patients (OA FLSs). FLSs were treated with IL-17 and their morphological features, respiratory level and mitochondrial gene expression were measured. The effects of IL-17 and Th17 cells on the relationship between autophagy and apoptosis were evaluated by measuring the expression of apoptosis-related genes using sodium nitroprusside or 3-methyladenine. The mitochondria of FLSs isolated from RA and osteoarthritis patients displayed different morphological and physiological features. RA FLSs exhibited greater autophagosome formation and greater dysfunction of mitochondrial respiration compared with OA FLSs. IL-17 induced mitochondrial dysfunction and autophagosome formation in RA FLSs, suggesting that they were resistant to apoptosis. Autophagy-related antiapoptosis induced by IL-17 was restored by inhibition of autophagy, suggesting a relationship between mitochondrial dysfunction and cell survival in RA FLSs. Th17 cells and IL-17 increased autophagy of RA FLSs by causing mitochondrial dysfunction. Our findings suggest that, in RA, interactions between RA FLSs and Th17 cells may be involved in the tumorous growth of FLSs and the formation of pannus in joints.

Cell Death and Disease (2017) 8, e2565; doi:10.1038/cddis.2016.490; published online 19 January 2017

Rheumatoid arthritis (RA) is the most common autoimmune disease and is characterized by progressive joint destruction and functional disability in affected people. The pathognomonic finding of RA is the expansive synovial tissue, called pannus, which erodes cartilage and bone at the cartilagebone interface. Fibroblast-like synoviocytes (FLSs) of the synovial lining can produce local inflammatory cytokines and proteolytic enzymes such as matrix metalloproteinases, which degrade the extracellular matrix. ${ }^{1}$ The pannus behaves like a locally invasive tumour, and the potential imbalance between the growth and death of RA FLSs is considered a target in the treatment of the disease.

Accumulating scientific evidence shows that interleukin 17 (IL-17) and IL-17-producing $\mathrm{T}$ helper (Th17) cells play critical roles during the development and progression of $\mathrm{RA}^{2,3}$ Although the exact pathogenesis of RA remains unclear, data from experimental models suggest that IL-17 plays a role in pannus growth, ${ }^{4}$ structural destruction of rheumatoid joints through receptor activator of nuclear factor kB ligand-independent osteoclastogenesis, ${ }^{5}$ and synovial neoangiogenesis. ${ }^{6}$

Mitochondrial dysfunction is emerging as a mechanism underlying various inflammatory and autoimmune diseases including cancer, atherosclerosis, neurodegenerative diseases, diabetes, obesity and autoimmune diseases. ${ }^{7-10}$ These diseases become worse when accompanied by systemic inflammation and oxidative stress. ${ }^{11}$ Mitochondria, indispensable organelles in mammalian cells, supply adenosine triphosphate (ATP), buffer calcium gradients and deposit unique molecules in cells. The mitochondrial respiratory machinery produces ATP by coupling oxygen and nutrients through oxidative phosphorylation (OxPhos); however, the exposure of cells to oxidative stress leads to mitochondrial damage, which stimulates their eventual elimination through autophagy. $^{12-14}$

Defective mitochondria are also degraded by autophagy, a process involving lysosomal degradation that restores homeostasis in multicellular organisms. Autophagy and autophagy proteins protect cells from cellular stressors such as viruses or bacteria, and control cell death, immune responses and inflammation. ${ }^{15-18}$ The two main self-destructive processes, autophagy and apoptosis, are closely related, and their interaction determines cell fate. Most of the processes of stress-related autophagy precede apoptosis, are mediated by caspase 6, caspase 7 and cytochrome c, and lead to the formation of apoptosomes and activated caspase 3. ${ }^{19,20}$ However, autophagy is known to inhibit indiscriminate cell

\footnotetext{
${ }^{1}$ The Rheumatism Research Center, Catholic Research Institute of Medical Science, The Catholic University of Korea, Seoul, South Korea; ${ }^{2}$ Laboratory of Immune Network, Catholic Research Institute of Medical Science, The Catholic University of Korea, Seocho-gu, Seoul, South Korea and ${ }^{3}$ Divison of Rheumatology, Department of Internal Medicine, The Catholic University of Korea, Seoul, South Korea

${ }^{*}$ Corresponding author: Professor M-L Cho, PhD, Rheumatism Research Center, Catholic Institutes of Medical Science, College of Medicine, The Catholic University of Korea, 505 Banpo-dong, Seocho-gu, Seoul 137-040, South Korea. Tel: +82-2-2258-7467; Fax: +82-2-599-4287; E-mail: iammila@ catholic.ac.kr

Received 19.8.16; revised 08.11.16; accepted 06.12.16; Edited by M Agostini.
} 
death to prevent unwarranted removal of functional mitochondria. 21,22

Autophagy plays a crucial role in immunity and inflammation. ${ }^{23}$ Excessive production of reactive oxygen species (ROS) by defective mitochondria may initiate inflammation. ${ }^{24}$ Mitochondrial oxidative stress caused by infection or inflammatory disease increases the secretion of chemokines and inflammatory cytokines, which stimulate infiltration by lymphocytes such as $\mathrm{T}$ helper (Th) cells. ${ }^{25}$ Mutations in mitochondrial genes are related to the pathogenesis and local inflammation involved in RA. ${ }^{26,27}$

We hypothesized that another pathological mechanism of mitochondrial dysfunction in Th17 cells is involved in RA. In the present study, we examined whether IL-17 can intensify RA severity by causing mitochondrial dysfunction. To test whether inflammation-related mitochondrial dysfunction is involved in RA, we studied FLSs isolated from patients with osteoarthritis (OA) or RA. We evaluated dysfunction of mitochondrial respiration, autophagy and autophagy-related apoptosis induced by IL-17 in these FLSs to examine further the relationship between inflammation, mitochondria and cell death in RA. All of our findings suggest that Th17 cells and IL-17 accelerate the autophagy-mediated antiapoptotic process in FLSs and contribute to the pathology of RA.

\section{Results}

Mitochondrial dysfunction is increased in RA FLSs. To investigate the mitochondrial dysfunction of RA FLSs, the morphologic changes of mitochondria were investigated in RA and OA FLSs isolated from synovia. Electron microscopy showed abnormal cristae in mitochondria and autophagosomes in both OA and RA FLSs. Interestingly, abnormal dark cristae in mitochondria were noted more frequently in RA FLSs compared with OA FLSs, which suggested a more abnormal mitochondrial function of RA FLSs ${ }^{28}$ (Figure 1a). MitoTracker staining showed significantly greater fragmentation and perinuclear clustering of mitochondria in RA FLSs compared with OA FLSs (Figure 1b). The distance of mitochondria from nuclei was shorter in RA FLSs than in OA FLSs.

These morphological differences observed in RA FLSs may imply mitochondrial dysfunction. Therefore, we next studied the functional impairment in mitochondria of RA FLSs. Confocal microscopy and flow cytometric analysis showed a significantly increased ratio of green to red staining in RA FLSs compared with OA FLSs, which indicated a lower mitochondrial membrane potential in RA FLSs (Figures 1c and d). The respiratory ability of mitochondria was also significantly lower in RA FLSs compared with that in OA FLSs (Figure 1e, upper panel). The basal, maximum and ATP-linked mitochondrial respiratory rates were all lower in RA FLSs than in OA FLSs (Figure 1e, lower panel).

Pathogenesis of RA is closely related to IL-17. Because IL-17-expressing effector T cells, called Th17 cells, are strongly implicated in RA pathogenesis, ${ }^{4,29}$ we studied the effects of IL-17 on mitochondrial function in RA FLSs. RA FLSs were incubated with IL-17 at a concentration of 2 or
$10 \mathrm{ng} / \mathrm{ml}$. MitoTracker staining showed that IL-17 treatment intensified the perinuclear clustering of mitochondria in RA FLSs (Figure 2a, left panel). The distance of mitochondria from nuclei was decreased by IL-17 treatment in a dosedependent manner (Figure $2 a$, right panel). The mitochondrial membrane potential was attenuated by IL-17 in RA FLSs (Figures $2 \mathrm{~b}$ and $\mathrm{c}$ ). Taken together, these findings suggest that IL-17 may be a critical pathological cytokine that impairs mitochondrial function in RA FLSs.

IL-17 disrupts mitochondrial respiration in RA FLSs. The main role of mitochondria is to produce ATP through respiration. We next examined whether IL-17 can cause changes in the composition or level of the respiratory machinery. Among the OxPhos complexes studied, the expression of complexes I and III was inhibited by IL-17 (Figure 3a). The basal and maximum respiratory levels, and reserve capacity in mitochondria, were also markedly reduced by $\mathrm{IL}-17$ (Figure $3 \mathrm{~b}$ ). These results suggested that each component of the respiratory apparatus was affected and contributed to the attenuation in mitochondrial respiratory function. Surprisingly, reverse transcription-quantitative polymerase chain reaction (RT-qPCR) results showed that IL-17 decreased the expression of the ATP- or respiration-related gene NDUFB5, a component of the respiration complex I; UQCRB, CYCS, COX5B and COX7B, components of respiration complex III; and ATP5O, a component of the F-type ATPase found in the mitochondrial matrix, in RA FLSs (Figure 3c). These data suggest that IL-17 decreases the expression of the genes for OxPhos complex components and that this may lead to impaired respiratory capacity in mitochondria of RA FLSs.

Next, we tried to identify the direct effects of Th17 cells on mitochondria of RA FLSs. RA FLSs were co-cultured with or without in vitro-differentiated human Th17 cells. Confocal microscopy showed that RA FLSs co-cultured with Th17 cells had a lower mitochondrial membrane potential compared with untreated cells (Figure 4a). Perinuclear clustering of mitochondria in RA FLSs increased after co-culture with Th17 cells (Figure 4b). The reserve respiratory capacity was not affected by co-culture with supernatant collected from Th17 cell or Th17 cells that had been co-cultured with FLS; however, the basal respiratory level, ATP-linked respiration and maximum respiratory level decreased markedly after co-culture with supernatant collected from Th17 cells or Th17 cells co-cultured with FLS (Figure 4c). The mRNA levels of OxPhos components were also decreased after co-culture of RA FLSs with Th17 cells (Figure 4d). This indicated that Th17 cells induced mitochondrial respiratory dysfunction in RA FLSs. Taken together, these data suggest that Th17 cells and the proinflammatory cytokine IL-17 cause both mitochondrial malformation and functional impairment of respiratory capacity and ATP production. We speculate that IL-17 may play a critical role in mitochondrial dysfunction in RA FLSs.

IL-17 stimulates autophagy by inducing mitochondrial dysfunction in RA FLSs. Damage caused by stressors such as ROS increases mitochondrial autophagy (also termed mitophagy). ${ }^{30}$ To study further the abovementioned results showing that $\mathrm{IL}-17$ and Th17 cells cause 
a

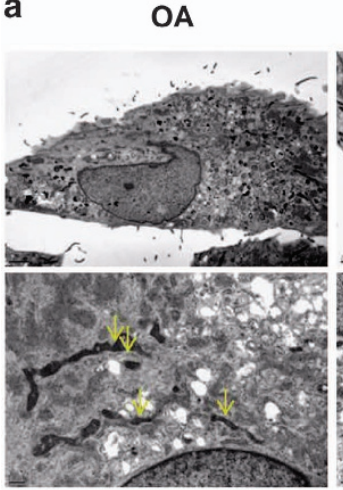

c
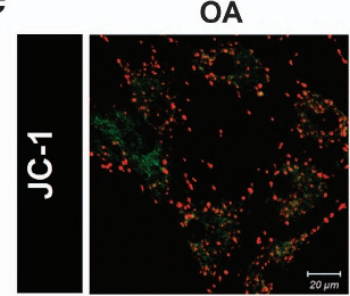

OA

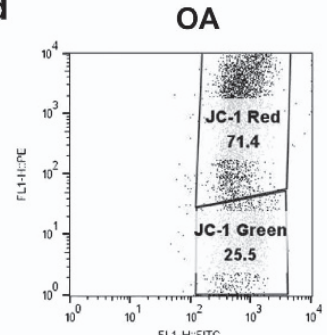

RA

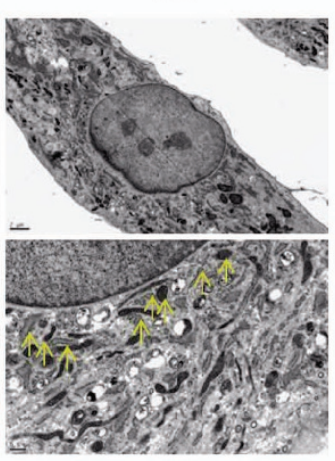

RA

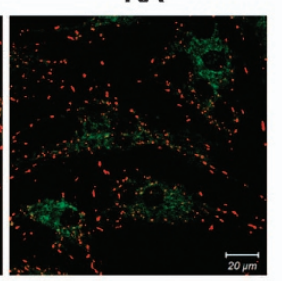

RA

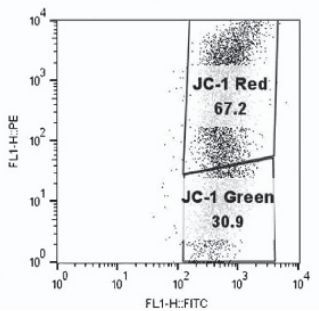

b
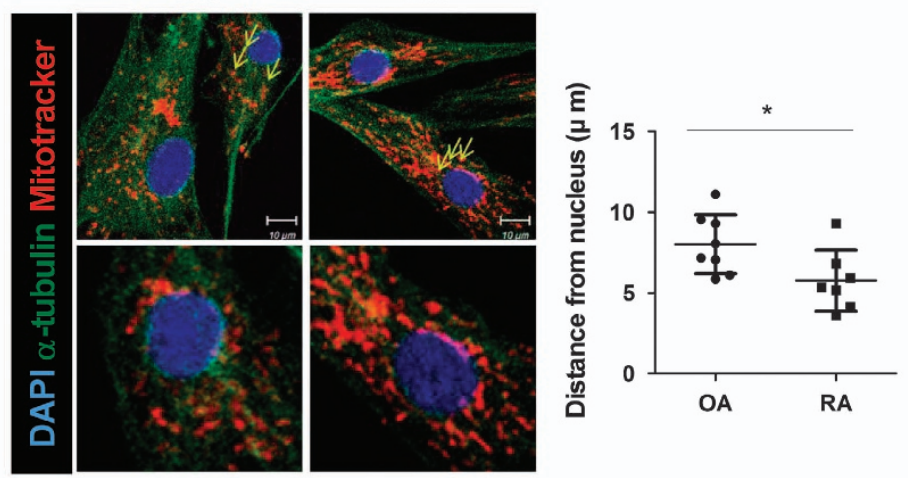

Figure 1 Mitochondrial dysfunction increases in RA FLSs. (a) Transmission electron microscopy images of FLSs from OA and RA patients. Arrowheads indicate mitochondria. Scale: $2 \mu \mathrm{m}$ (upper panels), $0.5 \mu \mathrm{m}$ (lower panels). (b) OA and RA FLSs were immunostained with MitoTracker Red CMXROS (red), anti- $\alpha$-tubulin (green), and DAPI (nuclei, blue). quantification of perinuclear mitochondrial distance in confocal micrographs of OA and RA FLSs. Data represent means \pm S.D. of five different culture experiments. The mitochondrial membrane potential was measured using JC-1 dye. (c) Confocal microscopy and (d) flow cytometry analysis. A representative density plot shows the OA and RA FLSs. (e) OCR in OA and RA FLSs (data are representative of three experiments). Quantification of mitochondrial respiration in OA and RA FLSs. The data are expressed as the mean \pm S.D. (or S.E.M.). ${ }^{*} P<0.05,{ }^{*} P<0.01,{ }^{* \star *} P<0.001$

mitochondrial dysfunction, we next examined the influence of IL-17 on autophagy in RA FLSs. FLSs were treated with or without IL-17 and autophagy was assessed. Electron microscopy showed that IL-17 treatment doubled the number of autophagosomes with dark cristae in RA FLSs (Figure 5a). This increase in autophagy was confirmed by the IL-17induced increase in staining for LC3, a marker of autophagy, in OA FLSs and RA FLSs. In addition, 3-methyladenine (3-MA), which inhibits autophagosome formation by inhibiting phosphoinositide 3-kinase, reduced LC3 expression (Figure 5b). Western blot analysis showed that the LC3-II/ LC3-I protein ratio increased from 0.94 in untreated cells to 1.12 after IL-17 treatment of RA FLSs. However, there was little change in this ratio in OA FLSs stimulated with IL-17. The 3-MA-treated FLSs showed decreased expression of LC3 compared with IL-17- or IL-17+3-MA-treated FLSs (Figure $5 \mathrm{c}$ ). We measured the mRNA levels of autophagy markers including ATG5 and $L C 3$ in OA FLSs and RA FLSs. IL-17 increased the expression levels of ATG5 and LC3 in RA
FLSs but caused few effects on the expression of these markers in OA FLSs (Figure 5d). Our data suggest that IL-17 can induce mitochondrial dysfunction, which results in autophagy and the abnormal expression of autophagyrelated genes in RA FLSs. In other words, IL-17-mediated inflammation seems to stimulate autophagy, which leads to mitochondrial dysfunction in RA FLSs.

Antiapoptosis is increased in RA FLSs by induction of IL-17-mediated autophagy. Autophagy is related closely to cell death because it induces apoptosis or necrosis-related proteins. ${ }^{18}$ We next evaluated the effects of $\mathrm{IL}-17$ on cell death in FLSs. As seen in Figure 6a, flow cytometric analysis showed that sodium nitroprusside (SNP) increased apoptosis-mediated cell death and reduced cell viability from $66.6 \%$ to $43.4 \%$. By contrast, IL-17 treatment restored cell viability to $55.7 \%$. The CCK-8 cell viability assay also showed that IL-17 protected cells from apoptosis caused by SNP and fully restored cell viability (Figure 6b). Immunocytochemical 

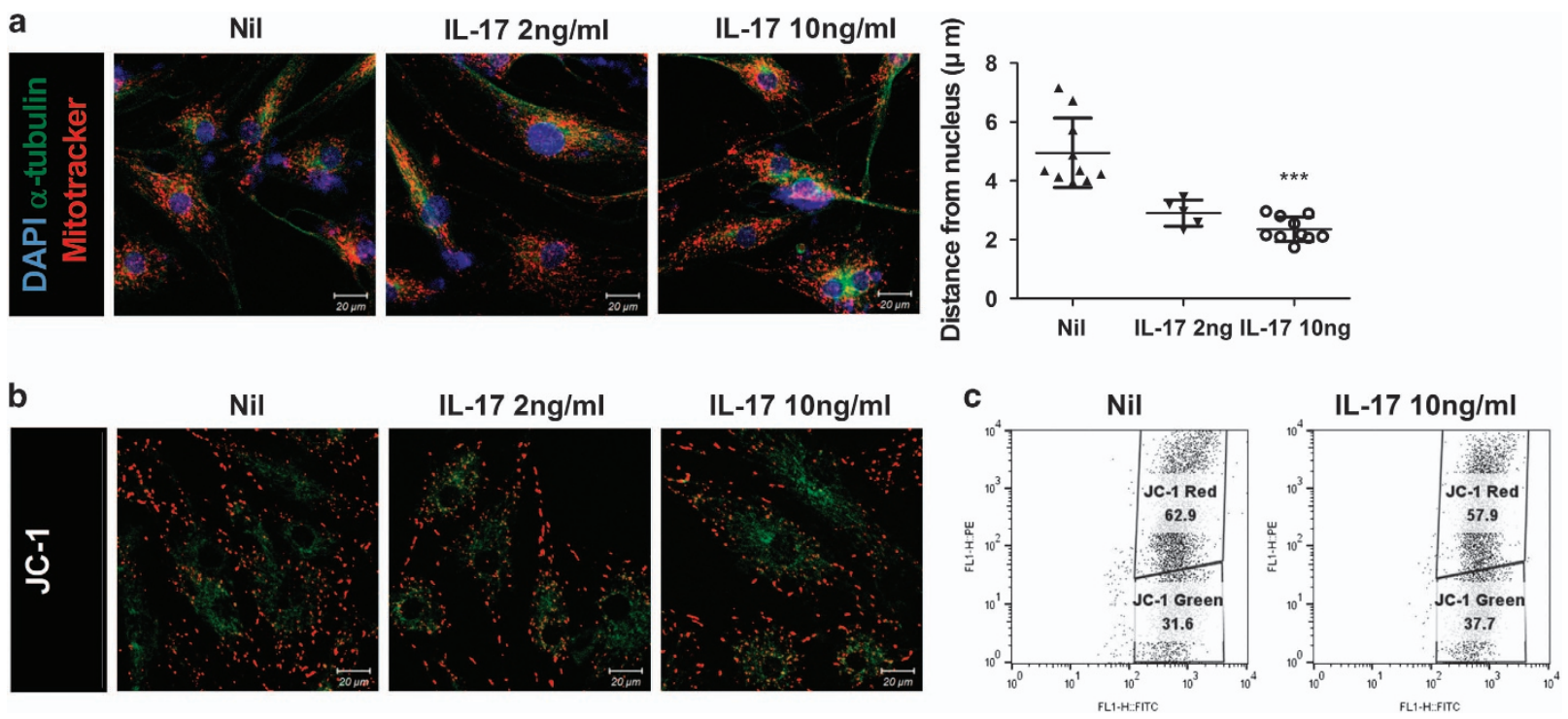

Figure 2 Pathogenesis of RA is mediated by IL-17. FLSs were cultured with IL-17 at concentrations of 0,2 or $10 \mathrm{ng} / \mathrm{ml} \mathrm{for} 12 \mathrm{~h}(n=5)$. (a) FLSs were immunostained with MitoTracker Red CMXROS (red), anti- $\alpha$-tubulin (green) and DAPI (nuclei, blue), and the perinuclear mitochondrial distance was quantified in confocal micrographs. A representative density plot shows the RA FLSs. Data represent the mean \pm S.D. ${ }^{* \star *} P<0.001$. Mitochondrial membrane potential was measured using JC-1 dye for confocal microscopy (b) or flow cytometry analysis (c)

a

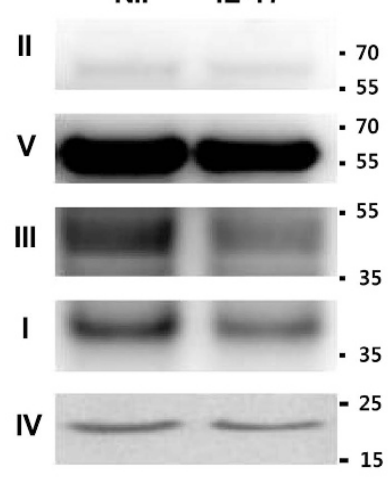

b

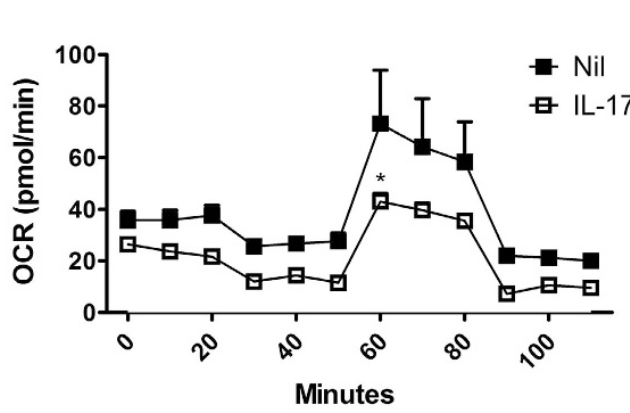

Basal respiration

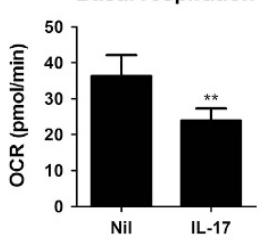

Maximal respiration

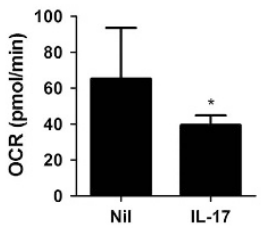

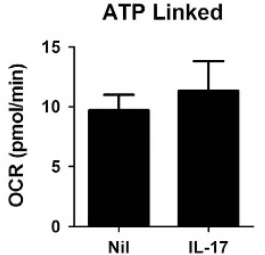

Reserve capacity

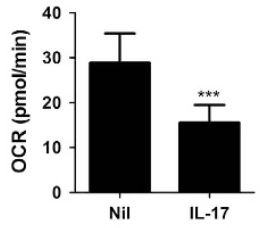

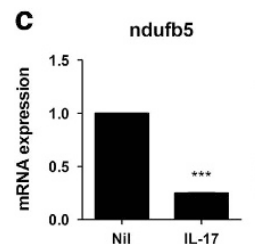
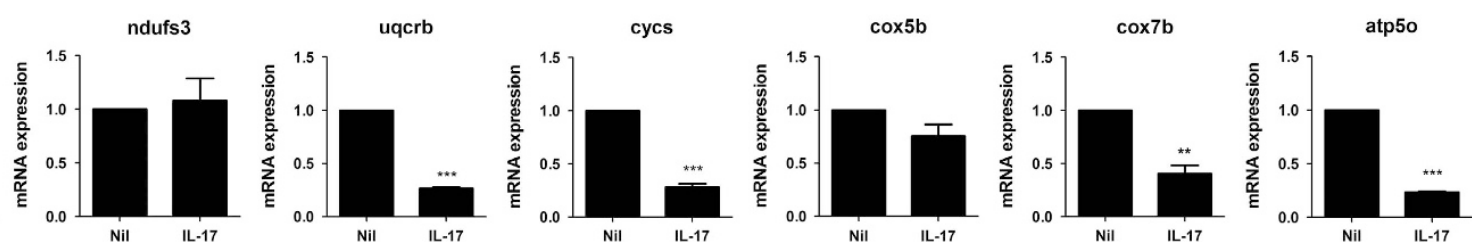

Figure 3 IL-17 disrupts mitochondrial respiration in RA FLSs. (a) The expression of OxPhos complex subunits in FLSs was analysed by western blotting. A representative figure is shown. (b) OCR in FLSs in the presence or absence of IL-17 (10 ng/ml). The data are representative of three independent experiments. Quantification of mitochondrial respiration in FLSs. (c) The expression level of mitochondrial OxPhos genes were determined using RT-qPCR in FLS incubated in the presence or absence of IL-17 (10 $\mathrm{ng} / \mathrm{ml}$ ) for 12 h. The data are expressed as the mean \pm S.D. (or S.E.M.). ${ }^{*} P<0.05,{ }^{* \star} P<0.01,{ }^{* \star} P<0.001$

staining indicated that IL-17 treatment increased the expression of the antiapoptotic protein BCL2 in RA FLS mitochondria (Figure 6c). However, IL-17 was unable to reverse the effects on cell death induced by 3-MA, which inhibits autophagosome formation by inhibiting phosphoinositide 3-kinase (Figure $6 \mathrm{~d}$ ). This suggests that the antiapoptotic function of IL-17 depends on autophagy.
Next, we tested whether the expression of apoptosis-related genes is dependent on IL-17 or autophagy. We performed RT-qPCR to examine the expression of genes for regulatory molecules of apoptosis such as BCL2 or caspase. The expression of the antiapoptotic protein BCL2 was increased sevenfold by IL-17 treatment compared with the control. The expression of proapoptotic molecules such as caspase 1 and 


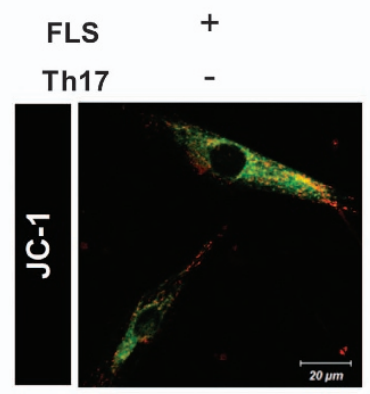

C

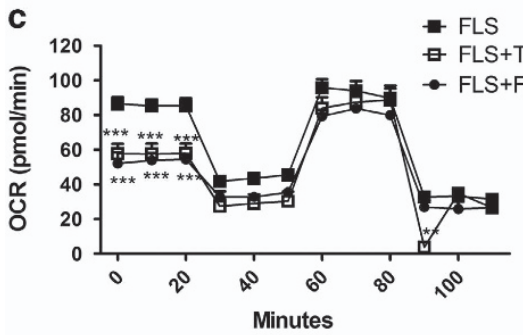

b

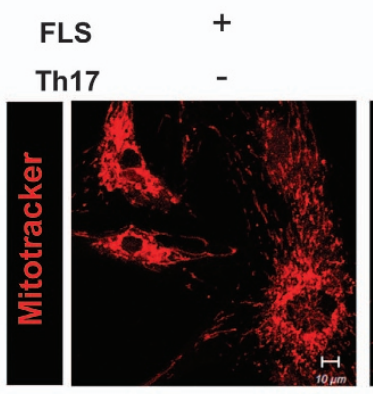

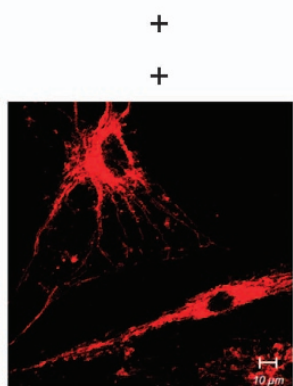

d
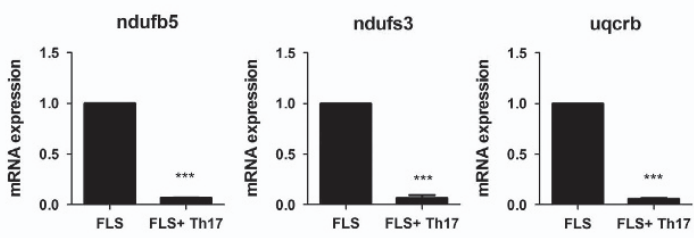

\section{- FLS}

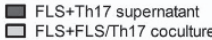
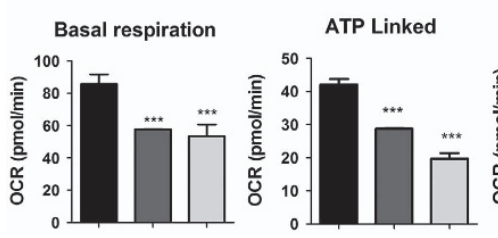
Maximal res:
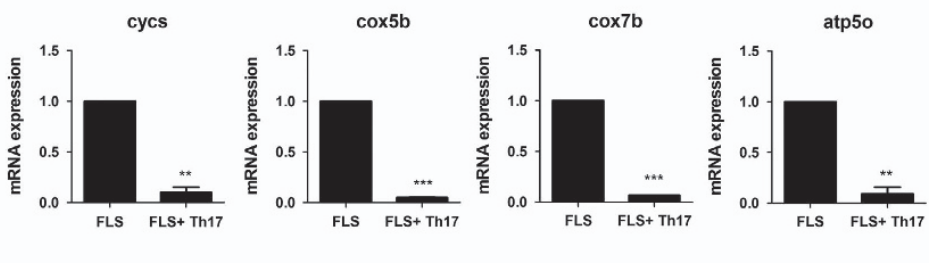

Figure 4 Th17 disrupts mitochondrial respiration in RA FLSs. FLSs isolated from RA patients were co-cultured with or without Th17 cells for $12 \mathrm{~h}$. (a) The mitochondrial membrane potential was measured using JC-1 dye and confocal microscopy. Green indicates decreased membrane potential. (b) Mitochondria were immunostained with MitoTracker Red CMXROS (red) and confocal microscopy images were captured. (c) The OCR was measured in FLSs co-cultured with or without supernatant collected from Th17 cells or from Th17 cells that had been co-cultured with FLS. Data are representative of three experiments. Quantification of mitochondrial respiration in RA FLSs. (d) Relative gene expression associated with mitochondrial OxPhos in RA FLSs. The data are expressed as the mean \pm S.D. (or S.E.M.). ${ }^{*} P<0.05$, ${ }^{* \star} P<0.01$, ${ }^{\star * *} P<0.001$

caspase 3 were reduced by IL-17 treatment (Figure 6e). Co-treatment with 3-MA and IL-17 reversed the effects of IL-17 on the expression of BCL2 and increased the expression of caspase 1 and caspase 3. Cytochrome c expression was also decreased by IL-17 and was recovered by 3-MA treatment (data not shown). These data indicate that IL-17 is a potent inhibitor of apoptosis by induction of autophagy in RA FLSs.

\section{Discussion}

In the present study, we investigated the possible mechanisms responsible for the effects of Th17 cells and IL-17 on mitochondrial dysfunction in RA FLSs. Although Th17 cells and IL-17 are known to be critical players during the RA inflammatory process, ${ }^{29,31,32}$ ours is the first study to show an association between IL-17- and Th17 cell-induced accelerated mitochondrial dysfunction and consequent induction of autophagy in RA FLSs. Our data suggest that mitochondrial malfunction in arthritis can be induced or augmented by IL-17 and/or Th17 cells.

We compared the functional differences in mitochondria between RA and OA FLSs. Although the destructive physiology of RA and OA is similar, the functions of mitochondria differ between these two diseases. The hallmarks of mitochondrial dysfunction, such as perinuclear clustering of mitochondria, abnormal dark cristae, autophagosome formation, and decreased membrane potential and respiration indicated that the inflammatory environment makes FLSs more vulnerable to mitochondrial malfunction. We also found that the distance of mitochondria from nuclei was shorter, which implies mitochondrial dysfunction, in RA FLSs than in OA FLSs (Figure 1a). Such asymmetric mitochondrial distribution may impair the capacity for ATP generation. ${ }^{33,34}$ This finding was confirmed by the lower respiration and membrane potential of mitochondria in RA FLSs (Figures 1c-e). The effects of inflammatory Th17 cells and IL-17 were more evident in RA FLSs. Treatment with IL-17, the main cause of $\mathrm{RA}$, intensified perinuclear clustering of mitochondria and decreased the membrane potential (Figure 2), a finding that was similar to the findings shown in Figure 1. Taken together, our data suggest that the FLSs accompanying inflammation in RA are more susceptible to attack by Th17 cells.

Morphological and physiological disorders of mitochondria reflect mitochondrial dysfunction and imply changes in the expression of components of the respiratory machinery. IL-17 and Th17 cells decreased the mitochondrial respiratory level and the protein levels of components of complex I, the largest complex in the respiratory chain, respiration complex III in the mitochondrial inner membrane and ATPase type-F component in the matrix (Figures 3 and 4). In our data, the effects of 
a

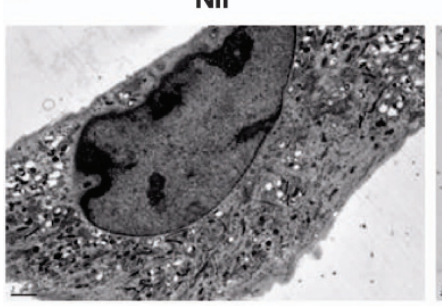

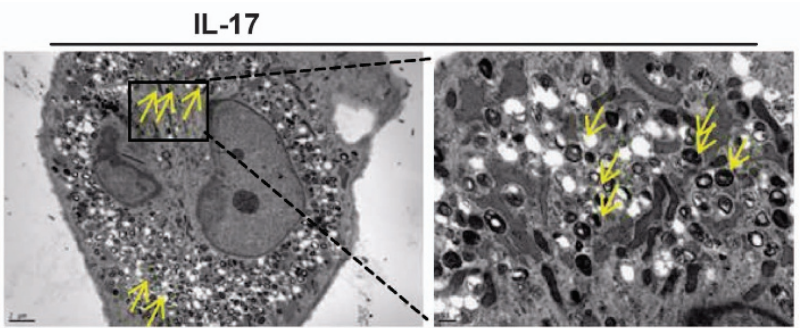

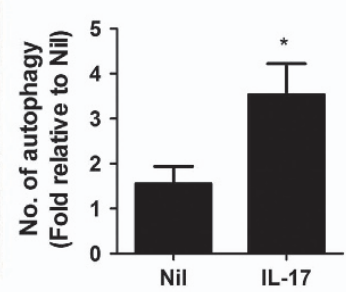

b

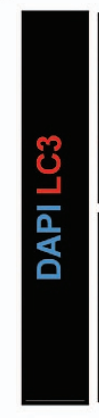

c

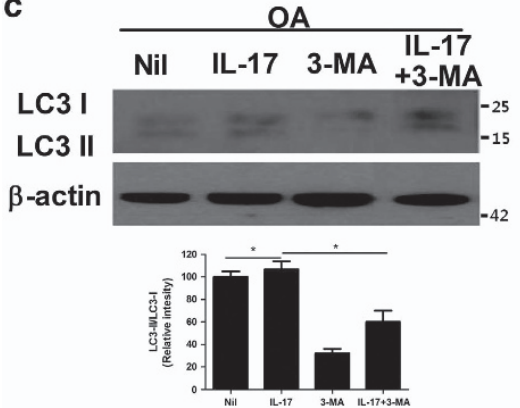

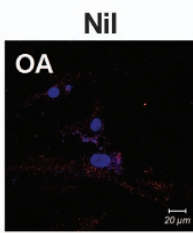

RA

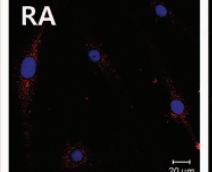

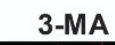
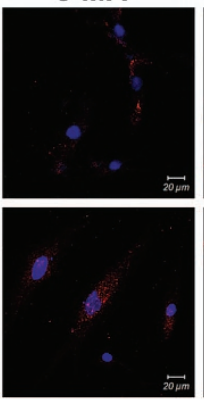
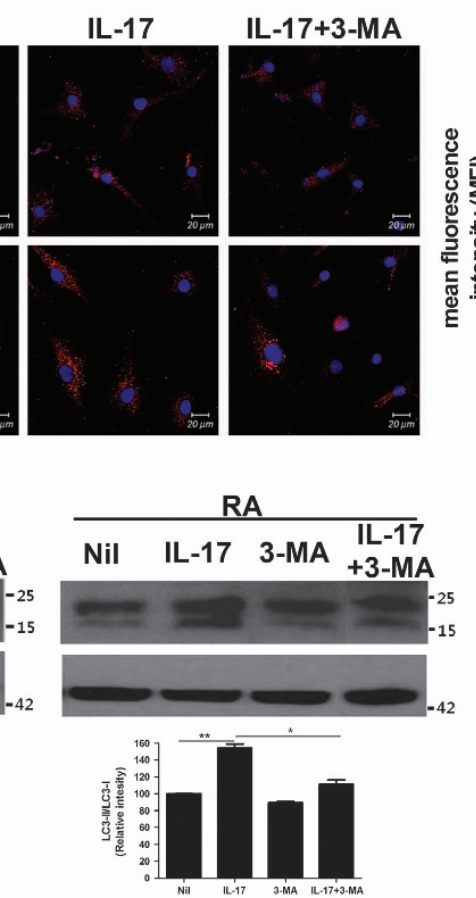

RA
$O A$

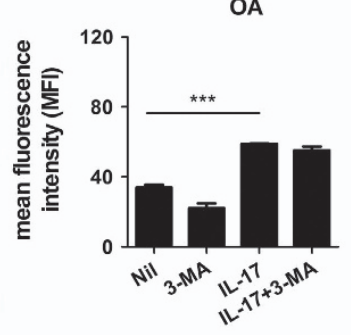

d

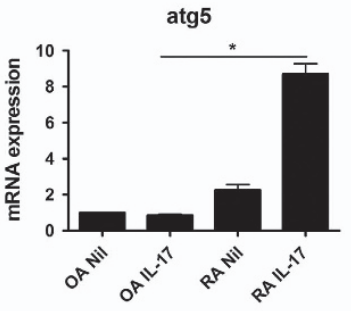

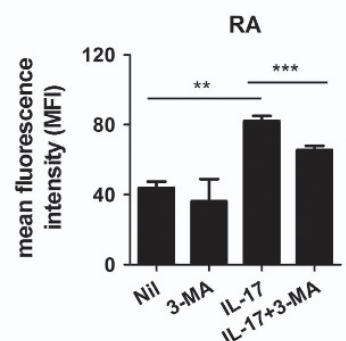

Ic3

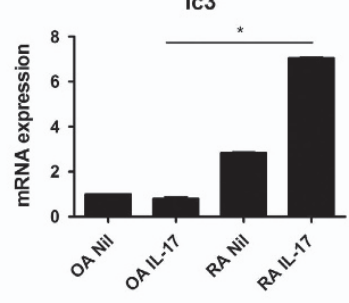

Figure 5 IL-17 induces autophagy by causing mitochondrial dysfunction in RA FLSs. (a) RA FLSs were cultured in the presence or absence of IL-17 (10 ng/ml) and transmission electron microscopy was performed. Arrowheads indicate autophagosomes. The right panels show magnified autophagosomes in RA FLSs cultured in the presence of IL-17. The number of autophagosomes in FLSs was quantified on the electron microscopy images. The data in the graph on the right are expressed as mean \pm S.D. of five different experiments. (b) OA FLSs and RA FLSs were immunostained for the autophagosome marker with anti-LC3 antibody and analysed by confocal microscopy. Scale: $20 \mu \mathrm{m}$. (c) Expression of LC3 in RA FLSs cultured with 3-MA (1 mM) in the presence or absence of IL-17 (10 ng/ml) was analysed by Western blotting. The LC3-I//LC3-I ratio was quantified. (d) The gene expression of ATG5 or LC3 associated with autophagy in FLSs cultured in the presence or absence of IL-17 (10 ng/ml) was quantified using RT-qPCR. The data are expressed as the mean \pm S.D. ${ }^{*} P<0.05,{ }^{* *} P<0.01,{ }^{* * *} P<0.001$

IL-17 were so significant that they disabled the main function of mitochondria, energy production by respiration. These destructive functions of IL-17 are related to the formation of autophagosomes. IL-17 treatment increased the expression of autophagy-specific markers such as LC3-I, LC3-II and ATG5 in RA FLSs, which also supports the idea that IL-17 plays a unique role in the differences between RA and OA (Figure 5).

In addition to causing malfunction of mitochondria, IL-17 or co-culture with Th17 cells also inhibited cell death. These effects were reversed by SNP, which increased apoptosis by affecting the expression of antiapoptotic or proapoptotic genes (Figures $6 \mathrm{a}$ and b). The expression of antiapoptotic genes such as BCL2 was increased markedly by IL-17, as we have previously reported. ${ }^{35}$ However, this antiapoptotic function of IL-17 was suppressed by inhibition of autophagy (Figures 6c and d). Co-treatment of FLSs with 3-MA and IL-17 showed clearly that antiapoptotic molecules and proapoptotic molecules are precisely regulated by autophagy and IL-17. We propose that autophagy is related to the mitochondrial malfunction caused by IL-17. In other words, inflammationrelated RA is susceptible to the effects of inflammation, inflammation increases autophagy-related gene expression and IL-17 accelerates these pathological mechanisms. Differentiated FLSs increase their deposition of collagen, resulting in synovial fibrosis. ${ }^{36}$ Therefore, the promotion of FLS survival by IL-17 may contribute to differentiation of FLSs and consequently, to synovial fibrosis.

Autophagy-related proteins beclin-1, ATG5 and LC3 are key players in the survival of FLSs in the early phase of RA or OA. ${ }^{37}$ However, FLSs undergo different cell fates in the late phases of RA and OA. In the early stage of bone destruction, beclin-1, LC3-II, ULK1 and ATG5 increase in both RA and OA FLSs, whereas their expression decreases in the late phase of 
a

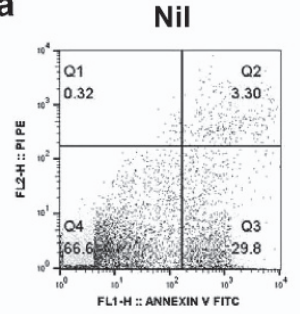

C

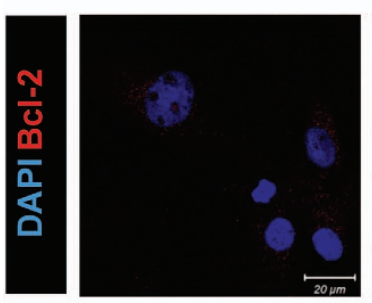

e

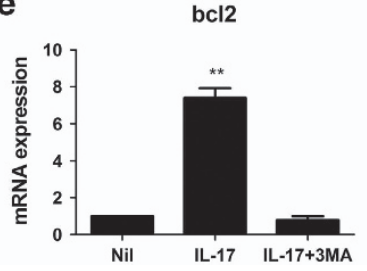

SNP

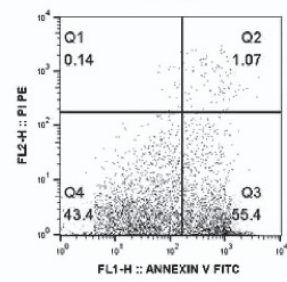

IL-17

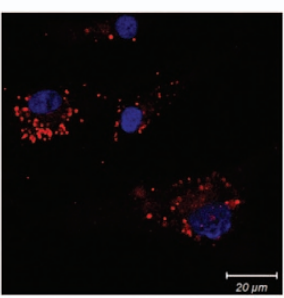

IL-17+SNP

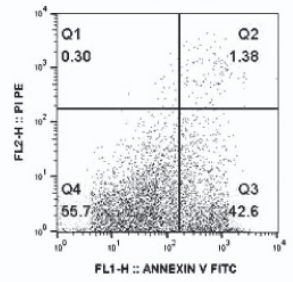

IL-17+3-MA

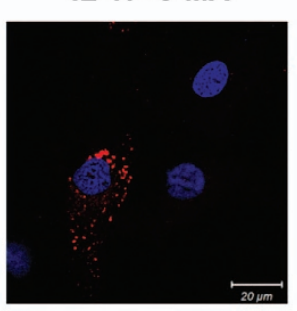

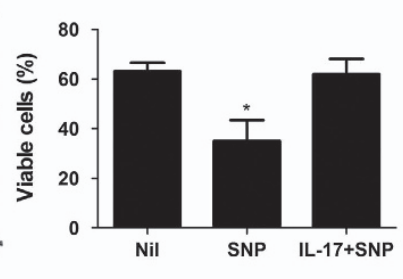

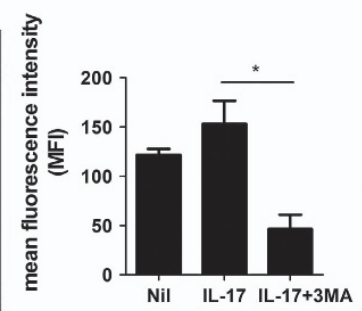

b

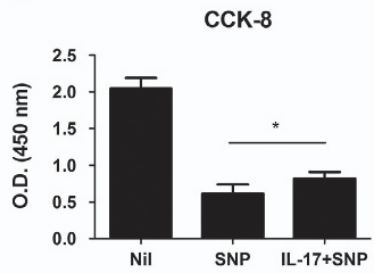

d

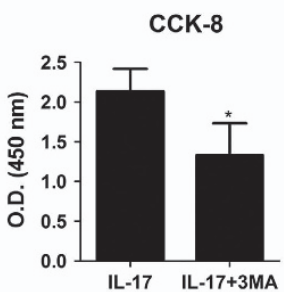

Figure 6 Induction of IL-17-mediated autophagy increases antiapoptosis in RA FLSs. (a) Apoptosis was induced by treatment of FLSs with 1 mM SNP. The degree of apoptosis was assessed by flow cytometry using propidium iodide and Annexin V staining. (c) Inhibition of autophagy with 3-MA decreased the expression of BCL-2 in RA FLSs in the presence of IL-17 (10 ng/ml). FLSs from RA were immunostained using anti-BCL2 antibody as the antiapoptosis marker and analysed by confocal microscopy. Scale: $20 \mu \mathrm{m}$. (b, d) The viability of synoviocytes were determined using a CCK-8 assay kit. (e) RT-qPCR was performed to quantify the relative expression of genes associated with apoptosis or antiapoptosis in RA FLSs cultured in the presence or absence of IL-17 (10 ng/ml) for $12 \mathrm{~h}$. The data are expressed as the mean \pm S.D. ${ }^{*} P<0.05,{ }^{* \star} P<0.01,{ }^{* \star *} P<0.001$

OA. ${ }^{38,39}$ Increased expression of beclin-1 and LC3 inhibits cell death in RA FLSs by stimulating autophagy. ${ }^{40}$ Antiapoptotic factors and autophagy increase, and proapoptotic factors decrease, which results in the survival of RA FLSs. ${ }^{40-43}$ By contrast, continued mechanical stress may decrease the abundance of ULK1, beclin-1 and LC3, which leads to cell death of chondrocytes in OA FLSs. ${ }^{44}$ However, IL-17 influences the expression of beclin-1 and promotes autophagy formation in B cells, ${ }^{45}$ which implies that IL-17 is important to the activation of autophagy in RA FLSs. Collectively, our findings suggest that an increased IL-17 level maintains autophagy, which allows the survival of RA FLSs. In other words, IL-17-mediated inflammation contributes to mitochondrial dysfunction and the resultant autophagy in RA FLSs, which subsequently stimulate the infiltration of inflammatory lymphocytic cells including Th17 cells.

Therefore, we suggest that a complex relationship exists between mitochondrial dysfunction, cell survival and IL-17induced autophagy in RA FLSs. This cooperation between FLSs and Th17 cells in RA seems to create a vicious positive feedback cycle of inflammation and an environment for survival of FLSs. We propose that collaboration between FLSs and Th17 cells may be one mechanism responsible for the tumorous growth of FLSs and formation of pannus in joints in RA.

\section{Materials and Methods}

Isolation and culture of human FLSs. Synoviocytes were isolated by enzymatic digestion of synovial tissue specimens obtained from patients with RA or $\mathrm{OA}$ undergoing total joint replacement surgery. The tissue samples were minced into 2- to 3-mm pieces and treated for $4 \mathrm{~h}$ with $4 \mathrm{mg} / \mathrm{ml}$ of type I collagenase (Sigma-Aldrich, St. Louis, MO, USA) in Dulbecco's modified Eagle's medium (DMEM) at $37{ }^{\circ} \mathrm{C}$ in $5 \% \mathrm{CO}_{2}$. Dissociated cells were centrifuged at $500 \mathrm{~g}$, resuspended in DMEM supplemented with $10 \%$ fetal bovine serum, $2 \mathrm{Mm}$ L-glutamine, $100 \mathrm{units} / \mathrm{ml}$ of penicillin, and $100 \mathrm{ng} / \mathrm{ml}$ streptomycin, and plated in $75-\mathrm{cm}^{2}$ flasks. After overnight culture, floating cells were removed, and the adherent cells were cultivated in DMEM supplemented with $10 \%$ fetal bovine serum. The cultures were kept at $37^{\circ} \mathrm{C}$ in $5 \% \mathrm{CO}_{2}$, and the medium was replaced every 3 days. The FLSs were passaged 3-8 times using trypsin-ethylenediaminetetraacetic acid (Gibco, Grand Island, NY, USA). The cells were seeded in six- or 24-well plates, eight-well chamber slides, or 100-mm culture dishes in $10 \%$ fetal bovine serumsupplemented DMEM, and then cultured for $12 \mathrm{~h}$ at $37^{\circ} \mathrm{C}$. FLSs were stained with allophycocyanin-conjugated anti-CD90 (eBioscience, San Diego, CA, USA) antibody as a fibroblast marker and analysed by flow cytometry. Informed consent was obtained from all participating subjects. The study received approval from the Institutional Review Board for Human Research, Seoul St. Mary's Hospital (KC14TISI0535).

Isolation and differentiation of peripheral blood mononuclear cells (PBMCs). Human PBMCs were isolated from heparinized blood samples using Ficoll-Hypaque (GE Healthcare Bioscience, Uppsala, Sweden) density gradient centrifugation. The isolated cells were cultured in RPMl1640 medium supplemented with $10 \%$ fetal bovine serum. To purify CD4+ T cells, PBMCs were incubated with CD4-coated magnetic beads and isolated using magnetic-activated cell sorting separation columns (Miltenyi Biotec, Bergisch Gladbach, Germany). Positively selected $\mathrm{CD} 4+\mathrm{T}$ cells were stimulated with plate-bound anti-CD3 
$(0.5 \mu \mathrm{g} / \mathrm{ml})$, soluble anti-CD28 $(1 \mu \mathrm{g} / \mathrm{ml}$; both from BD Biosciences, Sparks, MD, USA), anti-interferon- $\gamma(5 \mu \mathrm{g} / \mathrm{ml})$, anti-lL-4 $(5 \mu \mathrm{g} / \mathrm{ml})$, recombinant interleukin $1 \beta$

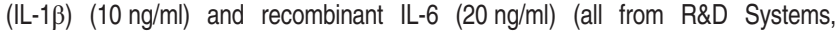
Minneapolis, MN, USA) for 3 days to achieve polarization of Th17 cells.

Co-culture of Th17 cells with RA FLSs. RA FLSs were seeded in 24-well plates at $1 \times 10^{6} /$ well in $10 \%$ DMEM. One day later, the cell culture medium was removed and the cells were cultured in $0.1 \%$ insulin-transferrin-selenium-A (ITSA)DMEM medium, then Th17 cells were layered onto the RA FLSs. Culture plates were incubated at $37^{\circ} \mathrm{C}$ for $24 \mathrm{~h}$, and the supernatants were collected.

Electron microscopy. Cells were fixed in $4 \%$ paraformaldehyde and $2.5 \%$ glutaraldehyde in $0.1 \mathrm{M}$ phosphate buffer overnight at $4{ }^{\circ} \mathrm{C}$. The cells were washed in $0.1 \mathrm{M}$ phosphate buffer, postixed with $1 \%$ osmium tetroxide for $1 \mathrm{~h}$ at $4{ }^{\circ} \mathrm{C}$ dehydrated through graded ethyl alcohol solutions, exchanged through acetone, and then embedded in Epon 812. Ultrathin sections $(70-80 \mathrm{~nm})$ were obtained on an ultramicrotome (Leica Ultracut, Leica, Vienna, Austria) and stained with uranyl acetate and lead citrate. Images were acquired at $60 \mathrm{kV}$ on a transmission electron microscope (JEM 1010, JEOL, Tokyo, Japan). The number of autophagosomes was counted manually. For each group, cells were randomly selected for the average number of autophagosomes/cell and the fold change in autophagosomes relative to the untreated control was plotted.

Analysis of mitochondrial membrane potential. The mitochondrial membrane potential was measured using JC-1 staining, confocal microscopy and flow cytometry. For JC-1 staining for confocal microscopy, $1 \times 10^{4}$ cells were cultured in $0.1 \%$ ITSA-DMEM medium in eight-well chamber slides in the presence or absence of IL-17 $(10 \mathrm{ng} / \mathrm{ml})$ for $12 \mathrm{~h}$. The cells were washed, the medium was changed to PBS, JC-1 dye was added, and the cells were incubated for $15 \mathrm{~min}$ at $37^{\circ} \mathrm{C}$. JC-1-labelled cells were washed in PBS and images were acquired on a confocal laser scanning microscope (LSM 510 Meta; Zeiss, Gottingen, Germany). For flow cytometric analysis of JC-1-stained cells, another $8 \times 10^{4}$ cells were cultured in six-well plates in the presence or absence of IL-17 $(10 \mathrm{ng} / \mathrm{ml})$ for $12 \mathrm{~h}$. The cells were harvested, JC-1 dye was added, and the cells were incubated for $15 \mathrm{~min}$ at $37^{\circ} \mathrm{C}$, washed, and analysed by flow cytometry on a FACSCalibur instrument (BD Biosciences). The fluorescence of the cells in each well was detected using 485-nm excitation wavelength and $530-\mathrm{nm}$ or $595-\mathrm{nm}$ emission wavelength.

MitoTracker staining and confocal laser scanning microscopy. For analysis of mitochondria using MitoTracker Red CMXROS (Molecular Probes, Eugene, OR, USA), the cells were seeded in eight-well chamber slides at a density of $1 \times 10^{4}$ cells/well in the presence or absence of IL-17 $(10 \mathrm{ng} / \mathrm{ml})$ for $12 \mathrm{~h}$. Mitochondria were stained with the MitoTracker Red probe for 30 min at $37^{\circ} \mathrm{C}$, after which the cells were washed with PBS, fixed with methanol, permeabilized with acetone, washed with PBS, and then blocked with $10 \%$ normal goat serum for $30 \mathrm{~min}$ at room temperature. The cells were stained at $4{ }^{\circ} \mathrm{C}$ overnight with monoclonal anti- $\alpha$-tubulin conjugate (Sigma-Aldrich) and nuclei were stained with 4',6-diamidino-2-phenylindole (DAPI). For measurement of the distance from the nucleus, the MitoTracker signal was divided into rings of equal area from the border of the nucleus. The signal for mitochondria was measured at the average distance within each area and these values were plotted. The cell sizes were measured in $\mu \mathrm{m}$ using LSM 510 Meta, Carl Zeiss software.

For confocal staining of LC3 or BCL2, cells were plated in eight-well chamber slides, washed with PBS, fixed with methanol $(10 \mathrm{~min})$, permeabilized with acetone (1 min), washed again with PBS, and then blocked with $10 \%$ normal goat serum for $30 \mathrm{~min}$. The cells were stained at $4^{\circ} \mathrm{C}$ overnight with anti-LC3 (Novus Biologicals, Littleton, CO, USA) or anti-BCL2 (Santa Cruz Biotechnology, Santa Cruz, CA, USA) antibodies. The primary antibody was detected with phycoerythrin-conjugated antirabbit IgG secondary antibody for $2 \mathrm{~h}$ at room temperature, and the nuclei were stained with DAPI. The stained cells were analysed using a confocal microscope (LSM 510 Meta; Zeiss). The expression of LC3 or BCL2 was estimated by comparing the mean fluorescence intensity using LSM 510 Meta, Carl Zeiss software.

Oxygen consumption rate (OCR). The XF24 Extracellular Flux Analyzer (Seahorse Bioscience, Chicopee, MA, USA) was used to measure the OCR. OA and RA FLSs were plated at $1.5 \times 10^{4}$ cells per well in XF24 well culture microplates. One day later, the cell culture medium was removed and the cells were cultured for $24 \mathrm{~h}$ in $250 \mu / /$ well of $0.1 \%$ ITSA-DMEM medium in 96 -well plates in the presence or absence of IL-17 $(10 \mathrm{ng} / \mathrm{ml})$ or the supernatant collected from Th17 cells or Th17 cells co-cultured with FLS. The culture medium was then removed, and the cells were washed and cultured in XF assay medium supplemented with $1 \mathrm{mM}$ sodium pyruvate, $2.5 \mathrm{mM}$ glucose and $4 \mathrm{mM}$ GlutaMax in a non- $\mathrm{CO}_{2}$ incubator. Mitochondrial electron transport was assessed through sequential injections of $4 \mu \mathrm{M}$ oligomycin, $3 \mu \mathrm{M}$ carbonyl cyanide 4-(trifluoromethoxy) phenylhydrazone (FCCP) and $2 \mu \mathrm{M}$ rotenone/2 $\mu \mathrm{M}$ antimycin A. Basal respiration was calculated as baseline OCR-rotenone/antimycin A OCR; ATP-linked respiration as basal respiration-oligomycin OCR; maximum respiration rate as FCCP OCR-rotenone/antimycin A OCR; and reserve capacity as maximal respiration-basal respiration.

Western blotting. The protein level of oxidative phosphorylation (OxPhos) complexes (I: $36 \mathrm{kDa} / 1621 \mathrm{bp}, \quad$ II: $70 \mathrm{kDa} / 2124 \mathrm{bp}, \quad$ III: $49.5 \mathrm{kDa} / 2226 \mathrm{bp}$, IV: $17 \mathrm{kDa} / 9219 \mathrm{bp}$ and V: $53 \mathrm{kDa} / 471 \mathrm{bp}$; Invitrogen, Carlsbad, CA, USA), LC3 (I: $19 \mathrm{kDa}$ and II: $17 \mathrm{kDa}$, Novus) and $\beta$-actin (Santa Cruz Biotechnology) was measured using a western blot system (SNAP i.d. Protein Detection System, Merck Millipore, Danvers, MD, USA). Cells were incubated in $100 \mathrm{~mm}$ dishes with 3-MA $(1 \mathrm{mM})$ in the presence or absence of IL-17 $(10 \mathrm{ng} / \mathrm{ml})$ for $12 \mathrm{~h}$, and mitochondrial and whole-cell lysates were prepared. The protein concentration was measured using the Bradford method (Bio-Rad, Hercules, CA, USA), and samples were separated on a $4-12 \%$ sodium dodecyl sulfate polyacrylamide gel and transferred to a nitrocellulose membrane (Amersham Pharmacia, Uppsala, Sweden). The primary antibodies to LC3, OxPhos complexes and $\beta$-actin were diluted in $0.1 \%$ skim milk in Tris-buffered saline Tween-20 and incubated for $15 \mathrm{~min}$ at room temperature. The membrane was washed and incubated with horseradish peroxidase-conjugated secondary antibody for $10 \mathrm{~min}$ at room temperature. Band density was estimated by image capture densitometry.

Mitochondrial fractionation. RA FLSs were incubated in the presence or absence of IL-17 $(10 \mathrm{ng} / \mathrm{ml})$ for $12 \mathrm{~h}$, and mitochondrial lysates were prepared. In brief, mitochondrial cell lysates were prepared from about $1 \times 10^{5}$ cells by homo-

Table 1 Primers used for real-time PCR

\begin{tabular}{|c|c|c|}
\hline Human genes & Forward & Reverse \\
\hline $\begin{array}{l}\text { NDUFB5 } \\
\text { NDUFS3 } \\
\text { UQCRB } \\
\text { CYCS } \\
\text { COX5B } \\
\text { COX7B } \\
\text { ATP5O } \\
\text { ATG5 } \\
\text { LC3 } \\
\text { BCL2 } \\
\text { CASPASE } 1 \\
\text { CASPASE } 3 \\
\text { GAPDH }\end{array}$ & $\begin{array}{l}\text { GCC ATG AGT TTG TTG CGG C } \\
\text { TGC TGT CTC TGT GTT CAA GG } \\
\text { GGA TGT TTC GCA TTA AGA GGG C } \\
\text { TGG GCC AAA TCT CCA TGG TC } \\
\text { AGT CCC CTC CAT CTC CAA CA } \\
\text { AGC CAC CAG AAA CGT ACA CC } \\
\text { GAG AGG TTC TCT CCC CTC ACT } \\
\text { GAC CTT CTG CAC TGT CCA TCT } \\
\text { CCG CAC CTT CGA ACA AAG AG } \\
\text { GGG GAG GAT TGT GGC CTT C } \\
\text { TTT CCG CAA GGT TCG ATT TTC A } \\
\text { ACA TGG CGT GTC ATA AAA TAC C } \\
\text { TGC CAA ATA TGA TGA CAT CA }\end{array}$ & $\begin{array}{l}\text { CCT TCG GAA AGC CAC GAG T } \\
\text { CTC GAA GCC ATA ATC TGT CAG G } \\
\text { TGG TCC ACT GCT CTT TAG GC } \\
\text { ATT GGC GGC TGT GTA AGA GT } \\
\text { CTG GGG CAC CAG CTT GTA AT } \\
\text { TAA CTC TGC CAA CAG GGG AC } \\
\text { CAA GGT ACC TCT CCG CGA TG } \\
\text { GCA ATC CCA TCC AGA GTT GC } \\
\text { AAG CTG CTT CTC ACC CTT GT } \\
\text { CAG GGC GAT GTT GTC CAC } \\
\text { GGC ATC TGC GCT CTA CCA TC } \\
\text { CAC AAA GCG ACT GGA TGA AC } \\
\text { GGA GTG GGT GTC GGT GTT G }\end{array}$ \\
\hline
\end{tabular}


genization in mitochondria fractionation buffer with $0.02 \%$ digitonin. The lysates were centrifuged at $900 \mathrm{~g}$ for $2 \mathrm{~min}$ at $4{ }^{\circ} \mathrm{C}$, and the supernatants were discarded. STM buffer: $250 \mathrm{mM}$ sucrose, $50 \mathrm{mM}$ Tris- $\mathrm{HCl}$ and $5 \mathrm{mM} \mathrm{MaCl}$ (STM) buffer was added to the pellets and pellets were centrifuged at $500 \mathrm{~g}$ for $15 \mathrm{~min}$ at $4{ }^{\circ} \mathrm{C}$. The supernatants were discarded, STM buffer was added again to the pellets, and the pellets were centrifuged at $1000 \mathrm{~g}$ for $15 \mathrm{~min}$ at $4^{\circ} \mathrm{C}$. STM supplemented with $1 \%$ NP-40 buffer was added to the pellets and the pellets were centrifuged at $1000 \mathrm{~g}$ for 5 min at $4{ }^{\circ} \mathrm{C}$. The resulting supernatants were used as the mitochondrial fraction.

Analysis of gene expression by reverse transcription-quantitative real-time PCR (RT-qPCR). Messenger RNA (mRNA) was extracted using TRIzol (Molecular Research Center, Cincinnati, OH, USA). cDNA was synthesized using the Superscript Reverse Transcription system (Takara, Shiga, Japan), then qPCR was performed using LightCycler FastStart DNA Master SYBR green I (Takara) following the manufacturer's instructions. All expression values were normalized to that of GAPDH mRNA. The primer sequences are described in Table 1.

Cell proliferation analysis. Cell proliferation was analysed using a CCK-8 kit (Dojindo Laboratories, Kumamoto, Japan) according to the manufacturer's instructions. Briefly, CCK-8 is reduced by dehydrogenases in cells to yield an orange-coloured product (formazan). ${ }^{46}$ The amount of formazan dye generated by the dehydrogenases is directly proportional to the number of living cells. RA FLSs $\left(5 \times 10^{3}\right.$ cells/well) were cultured in $200 \mu \mathrm{l}$ of $0.1 \%$ ITSA-DMEM medium in a 96-well plate with SNP $(1 \mathrm{mM})$ or 3-MA $(1 \mathrm{mM})$ in the presence or absence of IL-17 $(10 \mathrm{ng} / \mathrm{ml})$ for $12 \mathrm{~h}$. CCK-8 solution was added to each well, and the cells were incubated for $4 \mathrm{~h}$. Absorbance was measured at $450 \mathrm{~nm}$ on a microplate reader.

Annexin V and propidium iodide staining. RA FLSs were harvested, washed with PBS, and stained with fluorescein isothiocyanate-conjugated Annexin $\mathrm{V}$ (BD Biosciences) and propidium iodide (BD Biosciences) for $10 \mathrm{~min}$ at room temperature. The cells were subjected to flow cytometric analysis on a FACSCalibur instrument (BD Biosciences).

Statistical analysis. The results are expressed as mean \pm standard deviation (S.D.) or mean \pm standard error of the mean (S.E.M.). The data were analysed with Student's t-test or the Mann-Whitney $U$ test using Prism 5 software (GraphPad, La Jolla, CA, USA). $P<0.05$ (two-tailed) was considered significant.

\section{Conflict of Interest}

The authors declare no conflict of interest.

Acknowledgements. This research was supported by a grant of the Korea Health Technology R\&D Project through the Korea Health Industry Development Institute (KHIDI), funded by the Ministry of Health \& Welfare, Republic of Korea (grant number: H115C3062). This study was supported by a grant of the Korean Health Technology R\&D Project, Ministry for Health \& Welfare, Republic of Korea (HI14C3417). This research was supported by a grant of the Korea Health Technology R\&D Project through the Korea Health Industry Development Institute (KHIDI), funded by the Ministry of Health \& Welfare, Republic of Korea (HI15C1062).

1. Bartok B, Firestein GS. Fibroblast-like synoviocytes: key effector cells in rheumatoid arthritis. Immunol Rev 2010; 233: 233-255.

2. Chabaud M, Durand JM, Buchs N, Fossiez F, Page G, Frappart L et al. Human interleukin17: A T cell-derived proinflammatory cytokine produced by the rheumatoid synovium. Arthritis Rheum 1999; 42: 963-970.

3. Shen H, Goodall JC, Hill Gaston JS. Frequency and phenotype of peripheral blood Th17 cells in ankylosing spondylitis and rheumatoid arthritis. Arthritis Rheum 2009; 60: $1647-1656$.

4. Ito $\mathrm{H}$, Yamada H, Shibata TN, Mitomi H, Nomoto S, Ozaki S. Dual role of interleukin-17 in pannus growth and osteoclastogenesis in rheumatoid arthritis. Arthritis Res Ther 2011; 13: R14.

5. Sato K, Suematsu A, Okamoto K, Yamaguchi A, Morishita Y, Kadono Y et al. Th17 functions as an osteoclastogenic helper $\mathrm{T}$ cell subset that links $\mathrm{T}$ cell activation and bone destruction. J Exp Med 2006; 203: 2673-2682.

6. Pickens SR, Volin MV, Mandelin AM 2nd, Kolls JK, Pope RM, Shahrara S. IL-17 contributes to angiogenesis in rheumatoid arthritis. J Immunol 2010; 184: 3233-3241.
7. Medina-Gomez G. Mitochondria and endocrine function of adipose tissue. Best Pract Res Clin Endocrinol Metab 2012; 26: 791-804.

8. Pagano G, Castello G, Pallardo FV. Sjøgren's syndrome-associated oxidative stress and mitochondrial dysfunction: prospects for chemoprevention trials. Free Radic Res 2013; 47: 71-73.

9. Ouyang J, Wu M, Huang C, Cao L, Li G. Overexpression of oxidored-nitro domain containing protein 1 inhibits human nasopharyngeal carcinoma and cervical cancer cell proliferation and induces apoptosis: Involvement of mitochondrial apoptotic pathways. Oncol Rep 2013; 29: $79-86$.

10. Osellame LD, Blacker TS, Duchen MR. Cellular and molecular mechanisms of mitochondrial function. Best Pract Res Clin Endocrinol Metab 2012; 26: 711-723.

11. Hernandez-Aguilera A, Rull A, Rodriguez-Gallego E, Riera-Borrull M, Luciano-Mateo $F$, Camps $\mathrm{J}$ et al. Mitochondrial dysfunction: a basic mechanism in inflammation-related noncommunicable diseases and therapeutic opportunities. Mediators Inflamm 2013; 2013: 135698.

12. Westermann B. Bioenergetic role of mitochondrial fusion and fission. Biochim Biophys Acta 2012; 1817: 1833-1838.

13. Chang JC, Kou SJ, Lin WT, Liu CS. Regulatory role of mitochondria in oxidative stress and atherosclerosis. World J Cardiol 2010; 2: 150-159.

14. Chan DC. Mitochondria: dynamic organelles in disease, aging, and development. Cell 2006; 125: $1241-1252$.

15. Deretic V, Levine B. Autophagy, immunity, and microbial adaptations. Cell Host Microbe 2009; 5: 527-549.

16. Virgin HW, Levine B. Autophagy genes in immunity. Nat Immunol 2009; 10: 461-470.

17. Saitoh $T$, Akira $S$. Regulation of innate immune responses by autophagy-related proteins. $J$ Cell Biol 2010; 189: 925-935.

18. Marino G, Niso-Santano M, Baehrecke EH, Kroemer G. Self-consumption: the interplay of autophagy and apoptosis. Nat Rev Mol Cell Biol 2014; 15: 81-94.

19. Maiuri MC, Zalckvar E, Kimchi A, Kroemer G. Self-eating and self-killing: crosstalk between autophagy and apoptosis. Nat Rev Mol Cell Biol 2007; 8: 741-752.

20. Galluzzi L, Aaronson SA, Abrams J, Alnemri ES, Andrews DW, Baehrecke EH et al. Guidelines for the use and interpretation of assays for monitoring cell death in higher eukaryotes. Cell Death Differ 2009; 16: 1093-1107.

21. Kim I, Lemasters JJ. Mitophagy selectively degrades individual damaged mitochondria after photoirradiation. Antioxid Redox Signal 2011; 14: 1919-1928.

22. Grasso D, Ropolo A, Lo ReA, Boggio V, Molejon Ml, lovanna JL et al. Zymophagy, a novel selective autophagy pathway mediated by VMP1-USP9x-p62, prevents pancreatic cell death. J Biol Chem 2011; 286: 8308-8324.

23. Levine B, Mizushima N, Virgin HW. Autophagy in immunity and inflammation. Nature 2011; 469: 323-335

24. Witte ME, Geurts JJ, de Vries HE, van der Valk P, van Horssen J. Mitochondrial dysfunction: a potential link between neuroinflammation and neurodegeneration? Mitochondrion 2010; 10: $411-418$.

25. Liu TF, Brown CM, El Gazzar M, McPhail L, Millet $P$, Rao A et al. Fueling the flame: bioenergy couples metabolism and inflammation. J Leukoc Biol 2012; 92: 499-507.

26. Da Sylva TR, Connor A, Mburu Y, Keystone E, Wu GE. Somatic mutations in the mitochondria of rheumatoid arthritis synoviocytes. Arthritis Res Ther 2005; 7: R844-R851.

27. Harty LC, Biniecka M, O'Sullivan J, Fox E, Mulhall K, Veale DJ et al. Mitochondrial mutagenesis correlates with the local inflammatory environment in arthritis. Ann Rheum Dis 2012; 71: 582-588.

28. Sansanwal $P$, Yen B, Gahl WA, Ma Y, Ying L, Wong LJ et al. Mitochondrial autophagy promotes cellular injury in nephropathic cystinosis. J Am Soc Nephrol 2010; 21: 272-283.

29. van Hamburg JP, Asmawidjaja PS, Davelaar N, Mus AM, Colin EM, Hazes JM et al. Th17 cells, but not Th1 cells, from patients with early rheumatoid arthritis are potent inducers of matrix metalloproteinases and proinflammatory cytokines upon synovial fibroblast interaction, including autocrine interleukin-17 A production. Arthritis Rheum 2011; 63: 73-83.

30. Kroemer G, Marino G, Levine B. Autophagy and the integrated stress response. Mol Cell 2010; 40: 280-293.

31. Leipe J, Grunke M, Dechant C, Reindl C, Kerzendorf U, Schulze-Koops H et al. Role of Th17 cells in human autoimmune arthritis. Arthritis Rheum 2010; 62: 2876-2885.

32. Kim KW, Kim HR, Kim BM, Cho ML, Lee SH. Th17 cytokines regulate osteoclastogenesis in rheumatoid arthritis. Am J Pathol 2015; 185: 3011-3024.

33. Van Blerkom J, Davis P. Alexander S. Differential mitochondrial distribution in human pronuclear embryos leads to disproportionate inheritance between blastomeres: relationship to microtubular organization, ATP content and competence. Hum Reprod 2000; 15: 2621-2633.

34. Mizumura K, Cloonan SM, Nakahira K, Bhashyam AR, Cervo M, Kitada T et al. Mitophagydependent necroptosis contributes to the pathogenesis of COPD. J Clin Invest 2014; 124: 3987-4003.

35. Lee SY, Kwok SK, Son HJ, Ryu JG, Kim EK, Oh HJ et al. IL-17-mediated Bcl-2 expression regulates survival of fibroblast-like synoviocytes in rheumatoid arthritis through STAT3 activation. Arthritis Res Ther 2013; 15: R31.

36. Steenvoorden MM, Tolboom TC, van der Pluijm G, Lowik C, Visser CP, DeGroot J et al. Transition of healthy to diseased synovial tissue in rheumatoid arthritis is associated with gain of mesenchymal/fibrotic characteristics. Arthritis Res Ther 2006; 8: R165. 
37. Rockel JS, Kapoor M. Autophagy: controlling cell fate in rheumatic diseases. Nat Rev Rheumatol 2016; 12: 517-531.

38. Sasaki H, Takayama K, Matsushita T, Ishida K, Kubo S, Matsumoto T et al. Autophagy modulates osteoarthritis-related gene expression in human chondrocytes. Arthritis Rheum 2012; 64: 1920-1928.

39. Zhang Y, Vasheghani F, Li YH, Blati M, Simeone K, Fahmi H et al. Cartilage-specific deletion of mTOR upregulates autophagy and protects mice from osteoarthritis. Ann Rheum Dis 2015; 74: 1432-1440.

40. Xu K, Xu P, Yao JF, Zhang YG, Hou WK, Lu SM. Reduced apoptosis correlates with enhanced autophagy in synovial tissues of rheumatoid arthritis. Inflamm Res 2013; 62: 229-237.

41. Kato M, Ospelt C, Gay RE, Gay S, Klein K. Dual role of autophagy in stress-induced cell death in rheumatoid arthritis synovial fibroblasts. Arthritis Rheum 2014; 66: 40-48.

42. Shin YJ, Han SH, Kim DS, Lee GH, Yoo WH, Kang YM et al. Autophagy induction and CHOP under-expression promotes survival of fibroblasts from rheumatoid arthritis patients under endoplasmic reticulum stress. Arthritis Res Ther 2010; 12: R19.

43. Xu K, Cai YS, Lu SM, Li XL, Liu L, Li Z et al. Autophagy induction contributes to the resistance to methotrexate treatment in rheumatoid arthritis fibroblast-like synovial cells through high mobility group box chromosomal protein 1. Arthritis Res Ther 2015; 17: 374.

44. Carames B, Taniguchi N, Seino D, Blanco FJ, D'Lima D, Lotz M. Mechanical injury suppresses autophagy regulators and pharmacologic activation of autophagy results in chondroprotection. Arthritis Rheum 2012; 64: 1182-1192.
45. Yuan J, Yu M, Li HH, Long Q, Liang W, Wen S et al. Autophagy contributes to IL-17-induced plasma cell differentiation in experimental autoimmune myocarditis. Int Immunopharmacol 2014; 18: 98-105.

46. Hodgkin PD, Yamashita LC, Coffman RL, Kehry MR. Separation of events mediating B cell proliferation and lg production by using T cell membranes and lymphokines. J Immunol 1990; 145: 2025-2034.

cc (i) Cell Death and Disease is an open-access journal published by Nature Publishing Group. This work is

licensed under a Creative Commons Attribution 4.0 International License. The images or other third party material in this article are included in the article's Creative Commons license, unless indicated otherwise in the credit line; if the material is not included under the Creative Commons license, users will need to obtain permission from the license holder to reproduce the material. To view a copy of this license, visit http://creativecommons.org/licenses/by/4.0/

(C) The Author(s) 2017 